\title{
Manejo de complicación de fractura mandibular en un paciente pediátrico con dentición decidua
}

\author{
Management of a complicated mandibular fracture in a pediatric patient with primary dentition \\ Carlos Alvarado-Monge ${ }^{1, a}$, Helard Ventura-Ponce ${ }^{2, b}$, Ana Cecilia Ruiz-Imbert ${ }^{3, c}$, Emylú Miranda-Castillo ${ }^{4, b}$
}

\section{RESUMEN}

El trauma maxilofacial en la población pediátrica difiere del trauma en el adulto debido al potencial de crecimiento facial, el menor tamaño de los huesos, la mayor rapidez de regeneración de los tejidos, la presencia de dentición decidua o mixta y la dificultad en el manejo de conducta. Específicamente, el tratamiento de las fracturas mandibulares en niños es controversial y complicado porque puede tener un efecto negativo en el crecimiento mandibular, en la movilidad de la articulación temporomandibular y en el desarrollo y crecimiento de los gérmenes dentales, particularmente en la etapa de dentición decidua o mixta. Entre las opciones de tratamiento que reporta la literatura se incluyen el manejo conservador, el cerrado y el quirúrgico. El propósito del presente caso es relatar la complicación y manejo quirúrgico definitivo de la fractura mandibular desfavorable en un niño de 5 años de edad (dentición decidua) con material de osteosíntesis semirrígido no reabsorbible.

PALABRAS CLAVE: Fracturas mandibulares, preescolar, fijación interna de fracturas. (DeCS, Bireme)

Facultad de Estomatología Roberto Beltrán, Universidad Peruana Cayetano Heredia. Lima, Perú.

Asociación Peruana de Cirugía Bucal y Maxilofacial. Lima, Perú

Departamento de Ciencias Diagnósticas y Quirúrgicas, Facultad de Odontología, Universidad de Costa Rica. San José, Costa Rica.

Servicio de Cirugía Oral y Maxilofacial, Hospital Nacional Cayetano Heredia. Lima, Perú.

Residente de Programa de Especialización en Cirugía Oral y Maxilofacial.

Especialista en Cirugía Oral y Maxilofacial.

Especialista en Radiología Oral y Maxilofacial. 


\section{SUMMARY}

Maxillofacial trauma in the pediatric population differs from trauma in adults because of facial growth potential, smaller bones, faster tissue regeneration, deciduous or mixed dentition and the difficulties managing their behavior. Specifically, treatment of pediatric mandibular fractures is controversial and complicated because it can have a negative effect on mandibular growth, temporomandibular joint mobility, development and growth of tooth germs, particularly at the stage of deciduous or mixed dentition. Among the treatment options reported in the literature, conservative management, close reduction and surgery are included. The purpose of this report is to describe the complication and definitive surgical management of an unfavorable mandibular fracture in a five years old child, in primary dentition, with nonabsorbable material rigid osteosynthesis.

\section{KEYWORDS: mandibular fractures, child, internal fracture fixation. (MeSH, NLM)}

\section{INTRODUCCIÓN}

El trauma maxilofacial en pacientes pediátricos presenta diferencias en el manejo respecto a los adultos, constituyendo un reto para el planeamiento quirúrgico.

De todas las fracturas faciales, menos del 15\% se dan en la población pediátrica, siendo muy raras en menores de 5 años (1). Estas fracturas son menos frecuentes en niños que en adultos por varias causas. Una de ellas es la alta proporción cráneo:cara que presentan los niños (8:1 en el nacimiento, 4:1 a los 5 años y 2:1 en adultos), que permite que la fuerza del impacto sea absorbida por la frente y el cráneo en vez del rostro (2). En esta etapa, los niños son más propensos a sufrir lesiones cráneo-cerebrales, de tejido blando o trauma general de órganos (13). Conforme la edad avanza, el crecimiento facial se orienta hacia adelante y abajo, el tercio medio facial y la mandíbula se hacen más prominentes y la incidencia de fracturas faciales se incrementan (1). Otros factores que influyen en la baja frecuencia de fracturas faciales en niños son: la neumatización incompleta de los senos paranasales, la capa más gruesa de tejido adiposo, las suturas más elásticas y flexibles, la mayor cantidad de hueso esponjoso que cortical y la estabilización que proveen los dientes no erupcionados a los maxilares. Esto explica por qué las fracturas en tallo verde y condilares son más frecuentes en niños que en adultos.(4)

Las causas más frecuentes de trauma facial en niños son: caídas, lesiones asociadas a deportes y accidentes de tránsito. Además son más comunes en el género masculino en todos los grupos de edad, lo cual se atribuye a la preferencia de los varones por actividades más riesgosas (1).

Cuando sucede un trauma craneofacial en pacientes pediátricos, el impacto del mismo podría ser minimizado por el peso y la talla reducidos que presenta este grupo etario.(2)

Dentro de las fracturas de huesos faciales por trauma pediátrico, los huesos propios de la nariz son los más afectados, seguidos por el hueso mandibular (5), el mismo que se ve afectado en un tercio de los casos (2)

El objetivo del tratamiento es la restauración tridimensional precisa, funcional y estética, como antes de que sucediera la lesión (2); mediante una unión ósea que normalice la oclusión, restaure la forma y función, y no impida el crecimiento normal (6).

Varios factores hacen del tratamiento de las fracturas mandibulares en niños un tema controversial y complejo; por ejemplo, su efecto sobre el crecimiento mandibular, la movilidad de la articulación temporomandibular, el desarrollo y crecimiento de los gérmenes dentales, particularmente en la etapa de dentición mixta (7). El menor tamaño de los huesos, la mayor rapidez de regeneración de los tejidos y la dificultad en el manejo de la conducta, son también factores a considerar (8).

El manejo puede ser conservador con dieta blanda y observación, o bien mediante un abordaje operativo, como la reducción cerrada con fijación intermaxilar (FIM), el uso de férulas o splints, y la reducción 
abierta con fijación interna (RAFI) (4). Sin embargo, se prefiere la reducción cerrada con FIM debido a que las fracturas desplazadas son poco frecuentes y que los gérmenes dentarios pueden resultar dañados al utilizar material de osteosíntesis (9). En fracturas desplazadas desfavorables en niños, se puede considerar una reducción abierta con fijación interna (9). Sin embargo, estudios en animales muestran que la utilización de placas de fijación en el tercio medio facial puede retrasar el crecimiento, por lo que la fijación rígida debe de ser utilizada con precaución en niños y solo si otros medios de reducción y fijación no logran resultados aceptables (2).

$\mathrm{Si}$ se realiza una fijación rígida con placas y tornillos, estos deben colocarse en el borde inferior de la mandíbula para evitar dañar los gérmenes dentarios (2). También debe tomarse en cuenta que en niños el nervio alveolar inferior viaja adyacente a la cortical lingual cerca del borde inferior de la mandíbula, posicionándose en sentido cefálico conforme la edad y el tamaño de la mandíbula aumentan (4).

La información disponible es limitada en lo que respecta a la incidencia, características y manejo de las fracturas mandibulares de este grupo etario en el Perú.

El propósito del presente reporte es relatar el tratamiento definitivo, ante una complicación quirúrgica inicial, de una fractura mandibular desfavorable en un niño de 5 años de edad con dentición decidua.

\section{Reporte de caso}

Se reporta el caso de un niño de 5 años de edad, procedente de una zona rural del Departamento de Puno (Perú) que sufrió un accidente recreacional e ingresó cinco días después del trauma al Servicio de Cirugía Oral y Maxilofacial del Hospital Nacional Cayetano Heredia. De acuerdo al relato de la madre, el niño recibió el impacto de un marco metálico de futbol que cayó sobre su cara tras colgarse del mismo. La madre niega la ocurrencia de nauseas, vómito y pérdida de consciencia. El menor fue trasladado al centro de atención primaria más cercano, donde descartaron daño cerebral y/o cervical y fue derivado a la capital, Lima.

A la evaluación clínica, el niño se encontraba aprehensivo, no cooperador; presentaba una herida costrosa en la región del labio inferior lado derecho, tumefacción bilateral en la región geniana baja, alza térmica localizada, dolor a la palpación, recorrido condíleo conservado y cierre forzado de los labios (Figura 1.A). Intraoralmente presentaba una apertura bucal limitada de $1,5 \mathrm{~cm}$, maloclusión dental con mordida abierta anterior, fractura del segmento dentoalveolar maxilar superior desde la pieza 54 hasta la pieza 51, desplazado hacia lingual con exposición del ápice de las piezas 51 y 52, laceración gingival y una lesión de caries extensa en la cara vestibular de la pieza 53 (Figura 1.B).

La radiografía panorámica inicial muestra un trazo radiolúcido grueso, de trayectoria oblicua, proyectado en el hemimaxilar superior derecho, en la zona de piezas $54,53,52$ y 51, las mismas que se encuentran
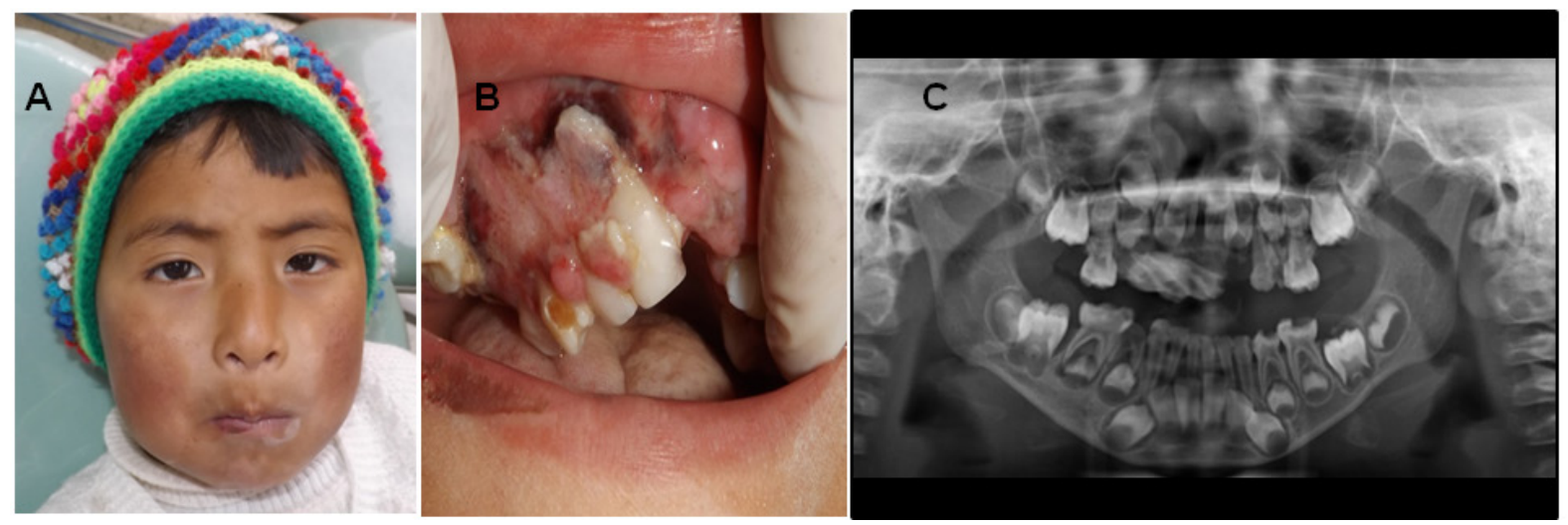

Figura1. Evaluación inicial. A: Cierre labial forzado. B: Fractura de segmento dento-alveolar I cuadrante. C: Radiografía panorámica inicial muestra fractura del segmento dentoalveolar en hemimaxilar superior derecho y ausencia de 61 y 62 . Además, encabalgamiento de segmentos y escalón en la basal mandibular en ángulo derecho, compatible con fractura mandibular. 
desplazadas; todos ellos, signos de fractura del reborde dentoalveolar maxilar. Asimismo, se observa la ausencia de las piezas dentarias 61 y 62, compatible con avulsión dentaria. En el ángulo mandibular derecho se observa una banda radiopaca producto del encabalgamiento de segmentos óseos y un escalón en la basal mandibular, signos radiográficos de fractura desplazada de cuerpo y ángulo mandibular derecho.

Por otro lado, a nivel del cuerpo mandibular izquierdo se observa una delgada línea radiolúcida que se extiende de manera oblicua desde la cima del reborde alveolar, por encima del germen de la pieza dentaria 36 , hasta la basal mandibular, que impresiona fractura mandibular del lado izquierdo. Además se evidencian múltiples lesiones cariosas, algunas de ellas con compromiso pulpar (Figura 1.C). Para una mejor evaluación de los hallazgos radiográficos, se realizó una tomografía espiral multicorte (TEM) con cortes de $1 \mathrm{~mm}$ de espesor, dicho examen diagnóstico se realizó bajó sedación consciente. El análisis tomográfico confirmó la presencia de fractura dentoalveolar desplazada palatinamente en el hemimaxilar superior derecho y disyunción maxilar a nivel de la línea media. También, la presencia de fractura mandibular desplazada en ángulo y cuerpo mandibular derecho y fractura mandibular no desplazada en el cuerpo mandibular izquierdo (Figura 2).

Diez días después del accidente, se realizó bajo anestesia general una reducción abierta con fijación interna (RAFI) mediante un abordaje intraoral y se colocó una miniplaca del sistema 2.0 con 4 tornillos monocorticales a nivel de cada trazo de fractura mandibular. Durante el evento quirúrgico se evidenció la presencia de un callo blando en los trazos de fracturas, el cual fue removido, dejando los

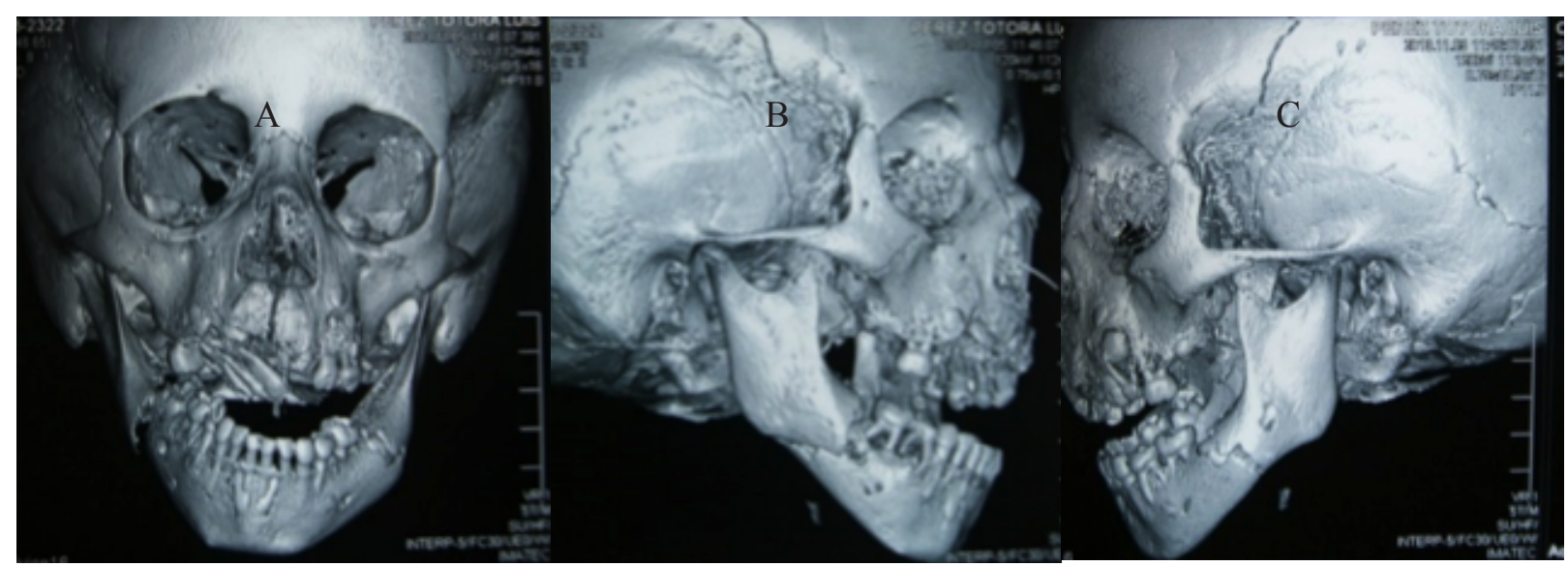

Figura 2. Tomografía Espiral Multicorte. Se observa, A: Fractura de segmento dento-alveolar en I cuadrante. B: fractura mandibular desplazada en ángulo-cuerpo derecho. C: Fractura no desplazada en cuerpo izquierdo.
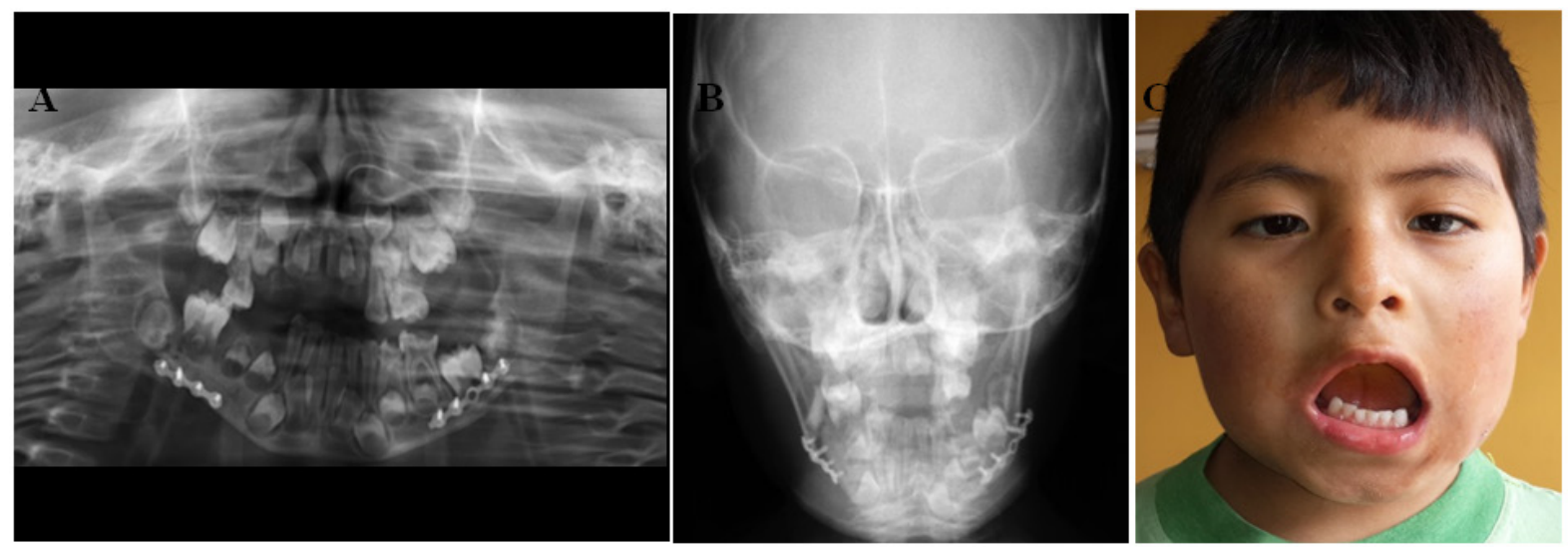

Figura 3. Control postquirúrgico luego de primera cirugía. A: Se observan escalones en la basal mandibular bilateral. B: Radiografía postero-anterior, se evidencia miniplacas desajustadas. C: Evaluación clínica 15 días después de la reducción abierta, se observa plano oclusal mandibular canteado. 
trazos limpios para una correcta reducción anatómica, esto debido a la falta de estabilidad oclusal. A nivel del maxilar superior, se realizaron las exodoncias de las piezas dentales 51, 52, 53 y 54, se reposicionó el segmento alveolar y se suturó la mucosa. Posteriormente se suministró tratamiento antibiótico endovenoso por 7 días (clindamicina, $40 \mathrm{mg} / \mathrm{kg}$ ).

El control radiográfico post-operatorio evidenció que la reducción de la fractura no fue satisfactoria, ya que en cada sitio de fractura mandibular se observaron escalones a nivel de la basal mandibular y el material de osteosíntesis no estaba bien conformado (Figura 3. A y B).

Seis días después de la primera intervención quirúrgica, la paciente presentó signos de flogosis con fluctuación a nivel de la región geniana baja del lado izquierdo, por lo que se realizó bajo anestesia general una incisión y drenaje de la secreción purulenta mediante un abordaje extraoral. Además se realizó la exodoncia de las piezas dentales 74 y 75 por presentar lesiones cariosas y se instauró nuevamente antibioticoterapia con clindamicina $40 \mathrm{mg} / \mathrm{kg}$ por 7 días más.

Una vez que la inflamación disminuyó y se consiguió la cooperación del niño, se evalúo clínicamente, evidenciándose un plano mandibular canteado con una latero desviación mandibular y apertura bucal hacia el lado derecho (Figura 3. C), razón por la cual 15 días después de la primera cirugía de reducción abierta se decidió reintervenir para sustituir las miniplacas por unas más largas y colocarlas a nivel de la basal mandibular. Para lograr este objetivo, se hizo necesario realizar un abordaje extraoral tipo Risdon bilateral (Figura 4).

En el control post-operatorio se solicitó una radiografía panorámica (Figura 5), una posteroanterior y una Towne inversa (Figura 6. A y B), en donde se evidenció la estabilidad y la reducción anatómica de los trazos de fractura, además de una buena conformación de las miniplacas. A la

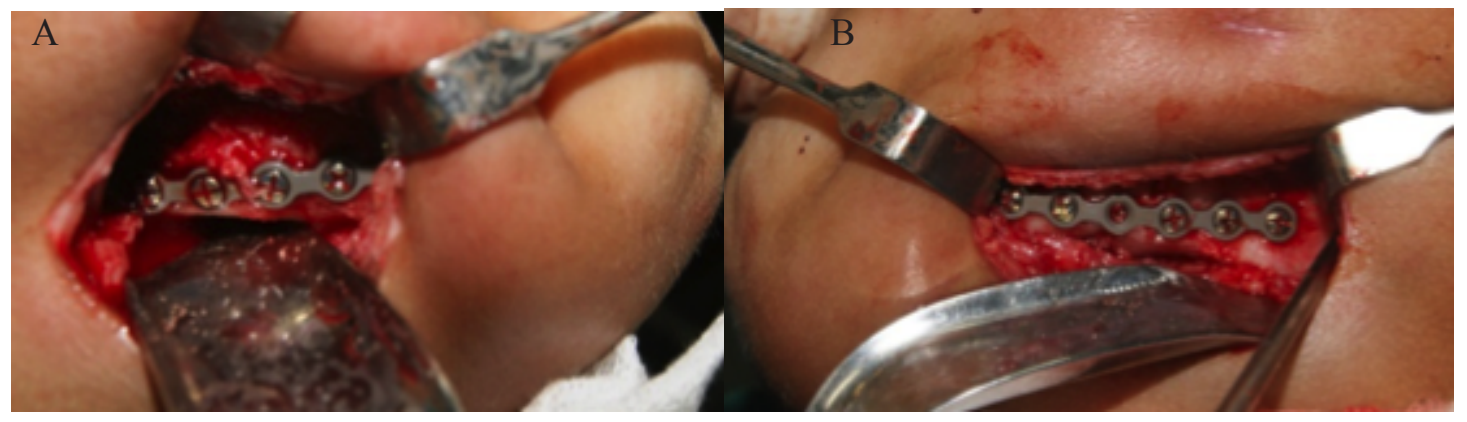

Figura 4. Segunda cirugía de reducción abierta. Abordaje extraoral tipo Risdon bilateral. A: Lado derecho. B: Lado izquierdo. Obsérvese el campo visual amplio y la colocación de las miniplacas de osteosíntesis del sistema 2.0 en la basal mandibular bilateral.

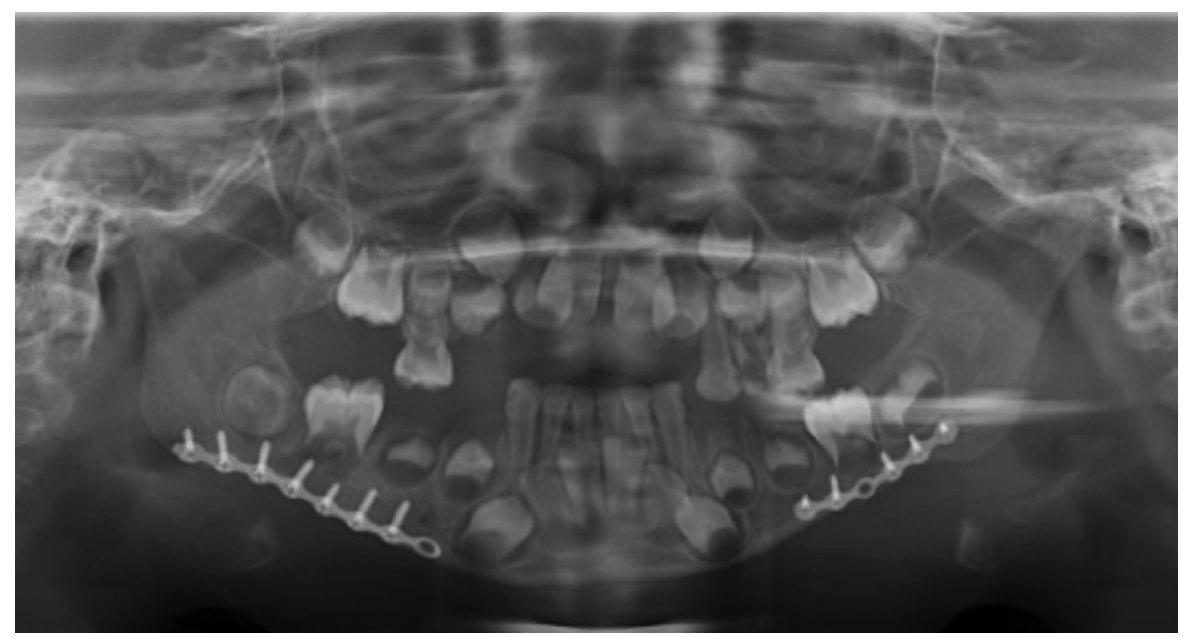

Figura 5. Control radiográfico luego de la segunda reducción abierta. Obsérvese la reducción anatómica y las miniplacas en la basal mandibular. 


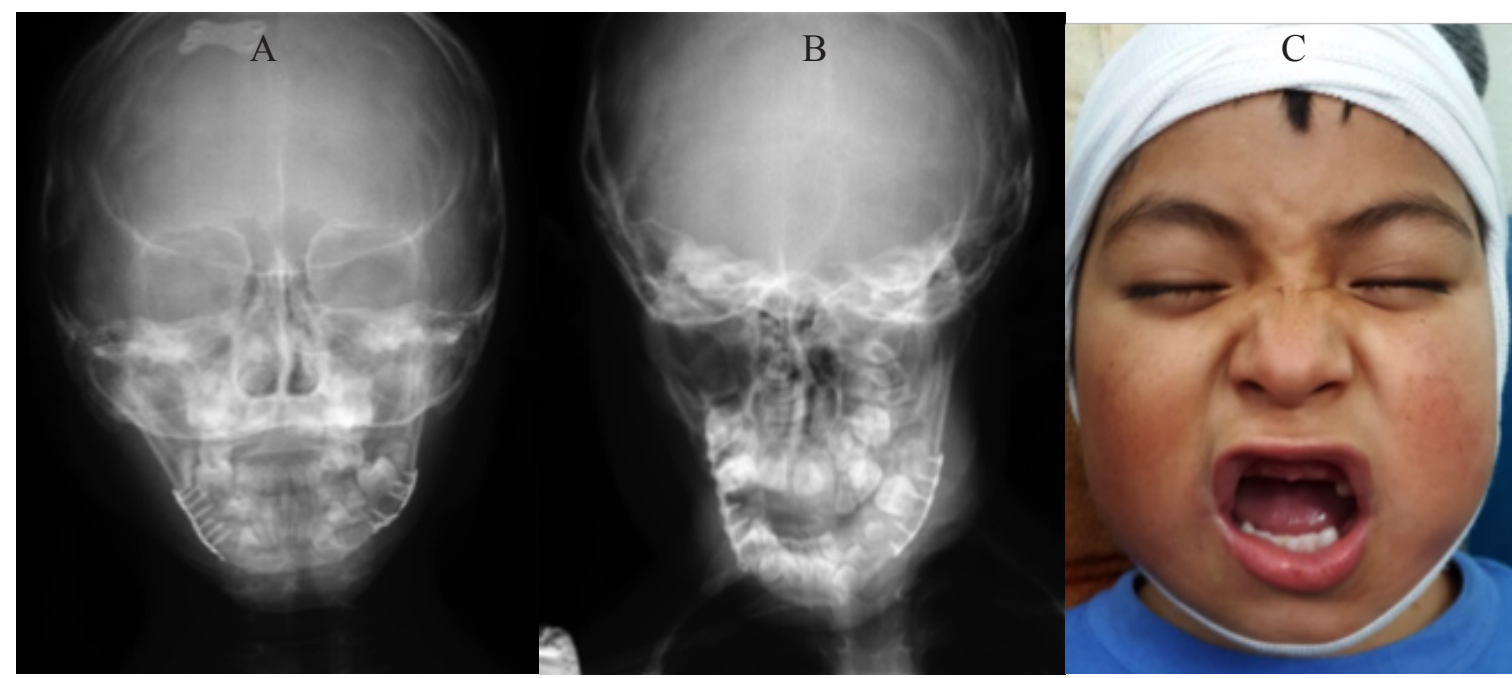

Figura 6. Control postoperatorio luego de la segunda reducción bierta. A: Radiografía postero-anterior. B: Radiografía Towne inversa. C. Control clínico 15 días después de la reintervención, obsérvese la recuperación del plano mandibular horizontal y apertura bucal.

evaluación clínica de seguimiento no se observó canteo en el plano mandibular y se recuperó la apertura bucal. (Figura 6. C).

\section{DISCUSIÓN}

El paciente pediátrico traumatizado puede resultar difícil de examinar clínicamente debido al dolor y a factores propios de la edad, por lo cual se hace indispensable un adecuado examen imaginológico (10). La adquisición de imágenes a un niño traumatizado despierto es muy compleja, por lo que generalmente se hace uso de la sedación consciente durante el procedimiento. Algunos autores sugieren que la radiografía panorámica, considerada el primer paso en la mayoría de los casos, puede ser obviada en niños pequeños, donde la modalidad de elección es la TEM (6). Sin duda, minimizar la dosis de radiación en un paciente en desarrollo es importante, pero en los casos donde hay compromiso del maxilar superior resulta difícil evaluar adecuadamente con una radiografía panorámica, debido a la superposición de estructuras anatómicas del tercio medio facial. Por esta razón en el caso reportado, se justificó la realización de una tomografía computarizada para una mejor evaluación del maxilar superior.

En los niños hay que tener en cuenta la inmensa capacidad de regeneración de los tejidos, el gran potencial osteogénico del periostio, la rica vascularización y el incremento metabólico; factores que propician una rápida consolidación de las fracturas faciales, por lo que deben ser reducidas lo más rápido posible para evitar una unión inadecuada (11).

La terap囚utica ideal de las fracturas maxilofaciales en ni冈os es aquel m凶todo m凶s simple que produzca una reducci冈n y estabilizaci $\bowtie n$ adecuadas (12).

Para la plani $\bigotimes_{c a c i \bigotimes n}$ del tratamiento, se deben tener en cuenta los siguientes factores: la localizaci $囚 n$ de la fractura, la edad del paciente, la etapa de dentici冈n (decidua, mixta o permanente) $(4,10)$, la estabilidad oclusal, la complejidad de la fractura, el tiempo transcurrido desde el trauma, las lesiones concomitantes y la experiencia del cirujano (12).

Las fracturas de cuerpo mandibular raramente afectan el crecimiento del hueso si el tratamiento se realiza de forma r囚pida y correcta (7); para lograr esto es necesario un tratamiento inicial temprano, adem凶s de experiencia en el manejo de fracturas en pacientes pedi区tricos.

Actualmente, existe debate sobre el tipo de reducci冈n $\mathrm{m} \bowtie s$ indicado en fracturas mandibulares en ni冈os. En general, la mayor de las fracturas mandibulares no desplazadas son manejadas conservadoramente, mediante una dieta blanda, analg凶sicos, precauciones en las actividades fखicas y controles peri冈dicos. Algunos casos pueden requerir

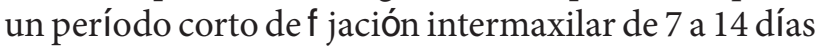
para reducir el dolor y corregir maloclusiones menores (4). Las maloclusiones mayores o desplazamientos

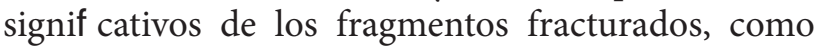


es el caso del presente reporte, requieren un abordaje $\mathrm{m} \rrbracket s$ complejo.

El éxito de la reducción cerrada con fijación intermaxilar depende de una oclusión dental sea estable que provea un soporte sólido. Desde el nacimiento hasta los 2 años de edad, hay poca dentición para inmovilizar la mandíbula. Entre los 2 años y los 4 años, la dentición decidua está bien formada y puede proveer soporte; de los 5 años a los 8 años, las molares deciduas pueden ser usadas para la inmovilización, mientras que los dientes anteriores están en varias etapas de exfoliación; de los 7 años a los 11 años, las molares permanentes e incisivos pueden ser utilizados para la FIM; después de los 11 años de edad la dentición presente soportará la FIM (12)

Cuando los métodos conservadores fracasan o cuando la reducción cerrada con FIM está imposibilitada por falta de soporte dental, se indica el uso de RAFI con el sistema de placas reabsorbibles (4). Las placas reabsorbibles son utilizadas actualmente en casos de craneosinostosis al igual que en traumas cráneo-maxilofacial con buenos resultados. Este material evita una segunda intervención para remover el material y hace que disminuya el uso de anestesia general (13). Sin embargo, el costo de este material representa una limitante para su uso en países latinoamericanos en vías de desarrollo, por lo que la opción de material de osteosíntesis de titanio generalmente resulta más viable.

Actualmente existe controversia respecto a la utilización de material de osteosíntesis no reabsorbible en niños; debido a la falta de estudios con seguimiento a largo plazo, no se ha consensuado que el titanio afecte el crecimiento mandibular, por lo que no hay lineamientos sobre si se debe remover la placa después de la consolidación de la fractura, decisión que muchas veces queda a criterio del cirujano (4).

Durante la colocación del material de osteosíntesis en las reducciones abiertas, el daño potencial a las raíces y los folículos dentales se puede minimizar con una técnica cuidadosa, en la cual se colocan tornillos bicorticales en el borde inferior de la mandíbula y tornillos monocorticales a nivel superior (13). La RAFI provee una reconstrucción tridimensional estable, promueve una cicatrización ósea primaria, disminuye el tiempo de tratamiento y elimina la necesidad de una FIM (4).

Otro punto importante en el manejo quirúrgico de las fracturas en niños es el tipo de abordaje, en este caso se realizaron dos tipos, en dos tiempos quirúrgicos diferentes. En la primera cirugía, se realizó un abordaje intraoral, presentándose las siguientes limitantes durante el acto operatorio:

- Campo visual disminuido, debido al tamaño de la cavidad bucal del paciente.

- Ausencia de estabilidad oclusal, debido a la etapa de dentición que presentaba el niño y la pérdida prematura de algunas molares deciduas.

- La longitud de las miniplacas de fijación resultó insuficiente, no permitían la adecuada inmovilización de los trazos de fractura.

Dichas circunstancias dificultaron la reducción anatómica y prolongaron el tiempo operatorio. Quince días después de la primera reducción, se realizó la reintervención quirúrgica mediante un abordaje extraoral, tipo Risdon bilateral. Así se logró tener una mejor visibilidad del campo operatorio, facilitando la colocación de las miniplacas a nivel del contorno de la basal mandibular; y permitiendo una reducción anatómica de la fractura. Para algunos clínicos esta vía no es la primera opción por la secuela de cicatriz facial y el posible daño a la rama marginal del nervio sétimo par craneal.

La etapa de dentición en la que se encontraba el paciente y la pérdida prematura de algunas piezas dentarias generó espacios edéntulos, por lo que luego de la reducción de los trazos fracturados quedaron pequeños márgenes de imperfección en la oclusión. Un estudio retrospectivo (7) que abarcó un período de 7 años e incluyó 19 casos de fracturas mandibulares en niños, mostró que 5 casos presentaron complicaciones, entre ellas: anquilosis de la ATM, desorden de la ATM y maloclusiones postoperatoria, siendo ésta última la dificultad que se presentó después de la primera RAFI en el presente caso.

Para descartar una anquilosis debido al trauma es necesario dar seguimiento por un periodo prolongado, al igual que para interceptar problemas en el crecimiento mandibular, el seguimiento ideal para el paciente es hasta que finalice el segundo pico de crecimiento. 
En el presente caso se utilizaron miniplacas de titanio del sistema 2.0, las cuales daban una buena estabilidad y una reducción anatómica. Algunos autores recomiendan retirar las placas 3 a 4 meses después de la consolidación de la fractura, mientras otros sugieren que se conserven para evitar una segunda intervención. Sin embargo, en el presente caso se planteó remover las placas después de 6 meses, en concordancia con el protocolo usado en la Universidad de Grecia (8).

El seguimiento a largo plazo es importante, ya que permitirá interceptar aberraciones durante el crecimiento debidas al trauma o al tratamiento realizado, posibilitando su manejo oportuno $(4,10)$.

\section{CONCLUSIONES}

La baja frecuencia de fracturas maxilofaciales en niños, en especial en centros hospitalarios no orientados exclusivamente a la atención pediátrica, dificulta el desarrollo de destrezas manuales en el abordaje quirúrgico de estos casos. Esto sumado a la falta de consenso en la literatura respecto al manejo ideal, hacen de estos casos un reto para el cirujano maxilofacial.

Se concluye que en pacientes pediátricos que presenten fracturas desfavorables del cuerpo y ángulo mandibular, donde con una RAFI con abordaje intraoral no se vaya a lograr una adecuada FIM intraoral, es recomendable realizar un abordaje extraoral para la colocación de la osteosíntesis, de manera que cumpla con los principios que promulga la Arbeitsgemeinschaft für Osteosynthesefragen (AO).

La controversia existente en la literatura con respecto al manejo de fracturas mandibulares en pacientes pediátricos, hace manifiesta la necesidad de realizar investigaciones prospectivas para posibilitar una toma de decisiones basada en evidencia.

\section{Correspondencia:}

\section{Carlos Ricardo Alvarado-Monge}

Av. Honorio Delgado 430, San Martín de Porres. Lima, Perú.

Correo electrónico: calvaradomonge@gmail.com

\section{REFERENCIAS BIBLIOGRÁFICAS}

1. Glazer M, Joshua BZ, Woldenberg Y, Bodner L. Mandibular fractures in children: analysis of 61 cases and review of the literature. Int $\mathrm{J}$ Pediatr Otorhinolaryngol. 2011; 75(1):62-4.

2. Abdullah WA. The use of a single titanium microplate in displaced pediatric parasymphysial mandibular fractures. Saudi Dent J. 2009; 21(2):95-100.

3. Zhou H-H, Ongodia D, Liu Q, Yang RT, Li ZB. Incidence and pattern of maxillofacial fractures in children and adolescents: a 10 years retrospective cohort study. Int J Pediatr Otorhinolaryngol. 2013; 77(4):494-8.

4. Wolfswinkel EM, Weathers WM, Wirthlin JO, Monson LA, Hollier LH, Khechoyan DY. Management of pediatric mandible fractures. Otolaryngol Clin North Am. 2013; 46(5):791-806.

5. Hegab A. Management of mandibular fractures in children with a split acrylic splint: a case series. Br J Oral Maxillofac Surg. 2012; 50(6):e93-e95.

6. Myall RWT. Management of mandibular fractures in Children. Oral Maxillofac Surg Clin N Am. 2009; 21(2):197-201.

7. Rémi M, Christine MC, Gael P, Soizick P, Joseph-André J. Mandibular fractures in children long term results. Int J Pediatr Otorhinolaryngol. 2003; 67(1):25-30.

8. Iatrou I, Theologie-Lygidakis N, Tzerbos F. Surgical protocols and outcome for the treatment of maxillofacial fractures in children: 9 years' experience. J CranioMaxillofac Surg. 2010; 38(7):511-6.

9. Kim TW, Seo EW, Song SI. Open reduction and internal fixation of mandibular fracture in an 11-monthold infant: a case report. J Korean Assoc Oral Maxillofac Surg. 2013; 39(2):90.

10. Zimmermann CE, Troulis MJ, Kaban LB. Pediatric facial fractures: recent advances in prevention, diagnosis and management. Int J Oral Maxillofac Surg. 2005; 34(8):823-33.

11. Kumaraswamy SV, Madan N, Keerthi R, Singh DS. Pediatric injuries in maxillofacial trauma: a 5 year study. J Maxillofac Oral Surg. 2009; 8(2):150-3.

12. Singhal R, Singh V, Bhagol A, Agrawal A, Kumar P. Pediatric maxillofacial injuries - If a new look is required? Int J Pediatr Otorhinolaryngol. 2013; 77(8):1333-6.

13. Marano R, de Oliveira Neto P, Sakugawa KO, Zanetti LSS, de Moraes M. Mandibular fractures in children under 3 years: a rare case report. Rev Port Estomatol Med Dentária E Cir Maxilofac. 2013; 54(3):166-70.

Recibido: 28/05/2014

Aceptado: 04/07/2014 